Supporting Information for the Manuscript Entitled

\title{
Formation of Ordered Mesoporous Films from In-situ Structure Inversion of Azo Polymer Colloidal Arrays
}

\author{
Yaobang Li, Xiaolan Tong, Yaning He, and Xiaogong Wang* \\ Department of Chemical Engineering, Laboratory for Advanced Materials \\ Tsinghua University, Beijing, 100084, P. R. China \\ E-mail Address $\square$ wxg-dce@mail.tsinghua.edu.cn
}




\section{Experiments}

Characterization. The TEM observation was carried out on a Hitachi H-800 transmission electron microscope with the acceleration voltage of $150 \mathrm{kV}$. The scanning electron microscope (SEM) measurement was performed on a field emission microscope JEOL JSM-6301F, which was operated with an accelerating voltage of $5 \mathrm{kV}$. All the samples prepared for SEM studies were coated with thin layers of carbon ( $\sim 5 \mathrm{~nm}$ in the thickness) before measurement. The atom force microscope (AFM) images were obtained by using a scanning probe microscope (Nanoscope IIIa) in the tapping mode. The sizes and size distribution of the colloidal spheres were measured with a Marvern Zetasizer 3000 dynamic light scattering instrument equipped with a multi- $\tau$ digital time correlation and a $632 \mathrm{~nm}$ solid-state laser light source. The scattering angle used for the measurement was $90^{\circ}$ and the temperature was controlled at $25^{\circ} \mathrm{C}$. The UV-Vis spectra of the samples were measured by using an Agilent $8453 \mathrm{UV}-\mathrm{Vis}$ spectrophotometer.

Laser irradiation setup. A linearly polarized beam from an $\mathrm{Ar}^{+}$laser at $488 \mathrm{~nm}$ was used as the light source. The spatially filtered laser beam was expanded and collimated. The intensity of the laser beam was about $150 \mathrm{~mW} / \mathrm{cm}^{2}$. The linearly polarized laser beam was incident perpendicularly to the wafer surfaces containing the colloids. The array of the ellipsoidal colloids was obtained by the irradiation of the laser beam for $10 \mathrm{~min}$. The light irradiation experiment was carried out at room temperature under an ambient condition. 


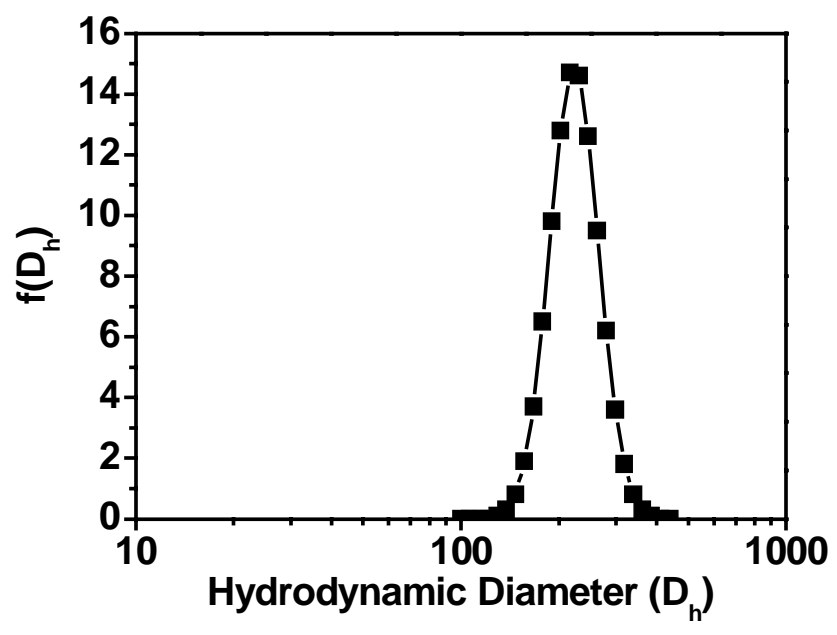

Figure S1: Hydrodynamic diameter and distribution of the colloidal spheres in water suspensions, measured by dynamic light scattering (DLS), the average $D_{\mathrm{h}}$ of spheres is $223 \mathrm{~nm}$ with the polydispersity index of 0.04 . 

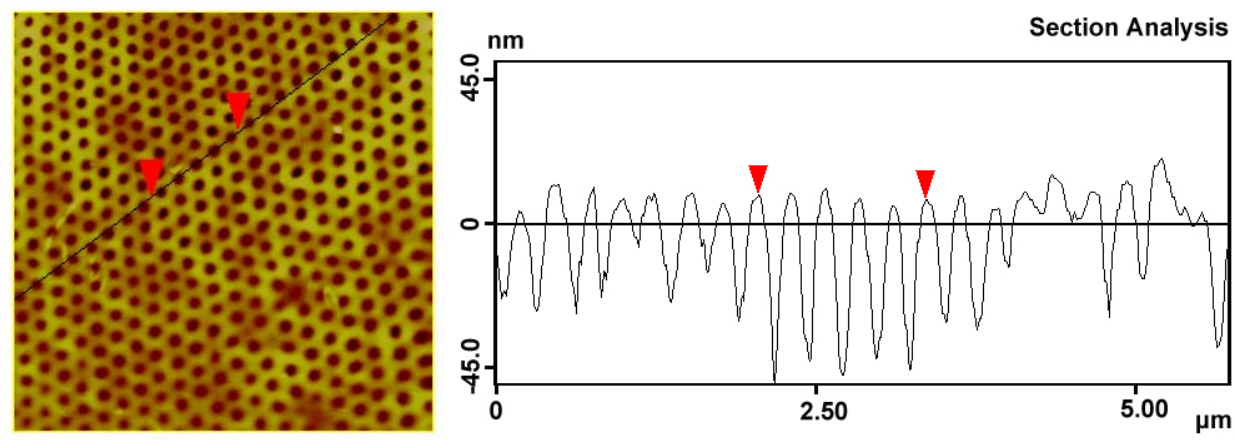

Figure S2: AFM 2D-view of the mesoporous film and the section analysis, the pore sizes and the distance between the pore centers were estimated to be $150 \mathrm{~nm}$ and $260 \mathrm{~nm}$ from the AFM section analysis. 


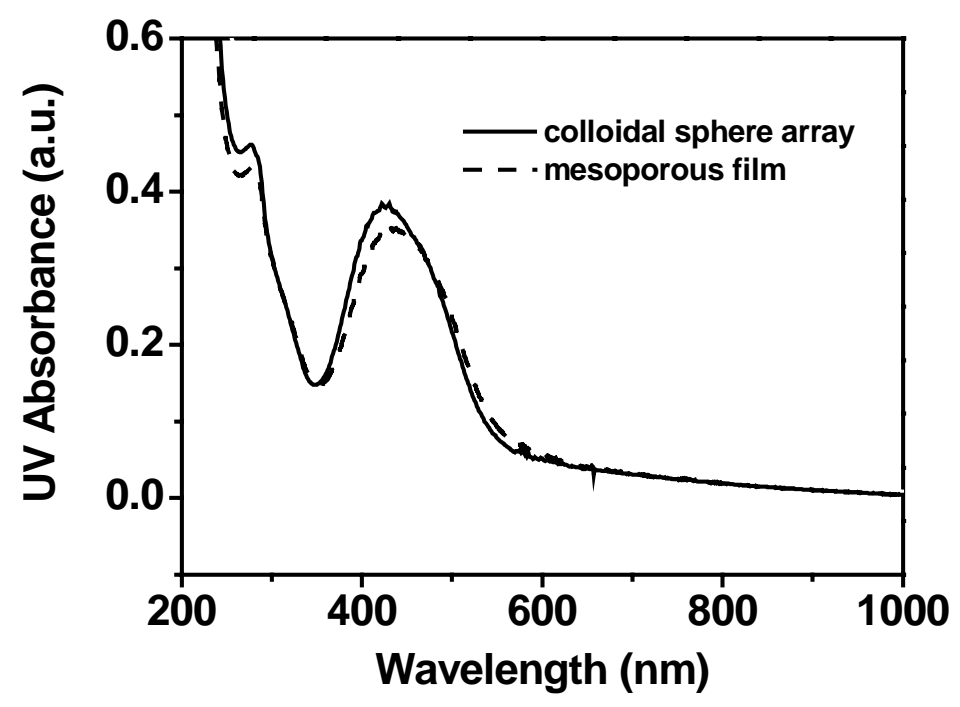

Figure S3: UV-Vis spectra of the two-dimensional colloidal sphere array and the mesoporous film obtained by the THF annealing. In the measurements, the light beam was incident vertically to the samples. The band at $430 \mathrm{~nm}$ is the characteristic absorption band ( $\pi-\pi^{*}$ transition) of BP-AZ-CA. 


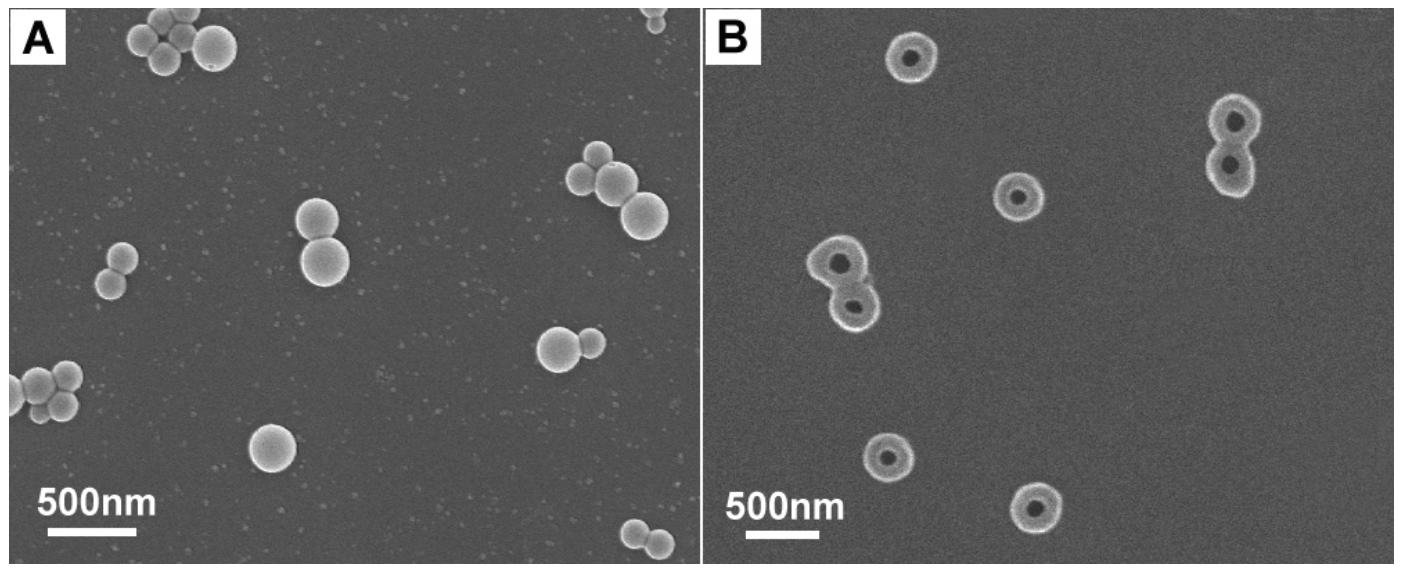

Figure S4: Typical SEM image of the 'isolated' spheres befor (A), and after (B) the THF treatment. 


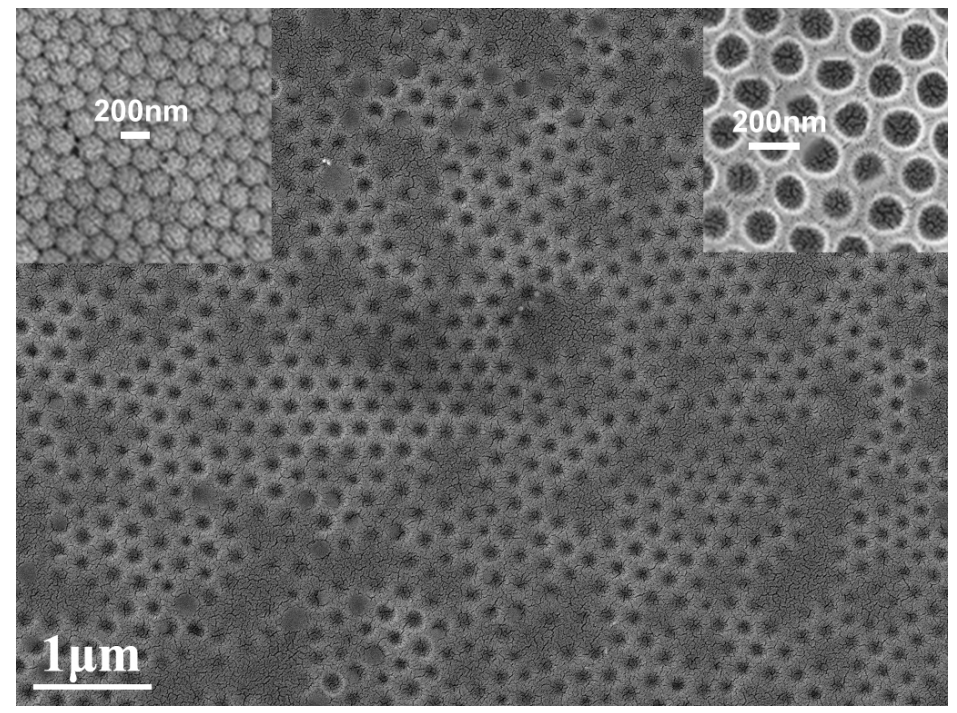

Figure S5: Typical SEM image of the porous structure formed by dropping THF on the colloidal array surface. The THF amount is $50 \mu \mathrm{THF}$ per $0.5 \mathrm{~cm}^{2}$. Inset (left): SEM image of the colloidal sphere arrays before the solvent treatment. Inset (right): enlarged SEM image of the mesoporous structure. 


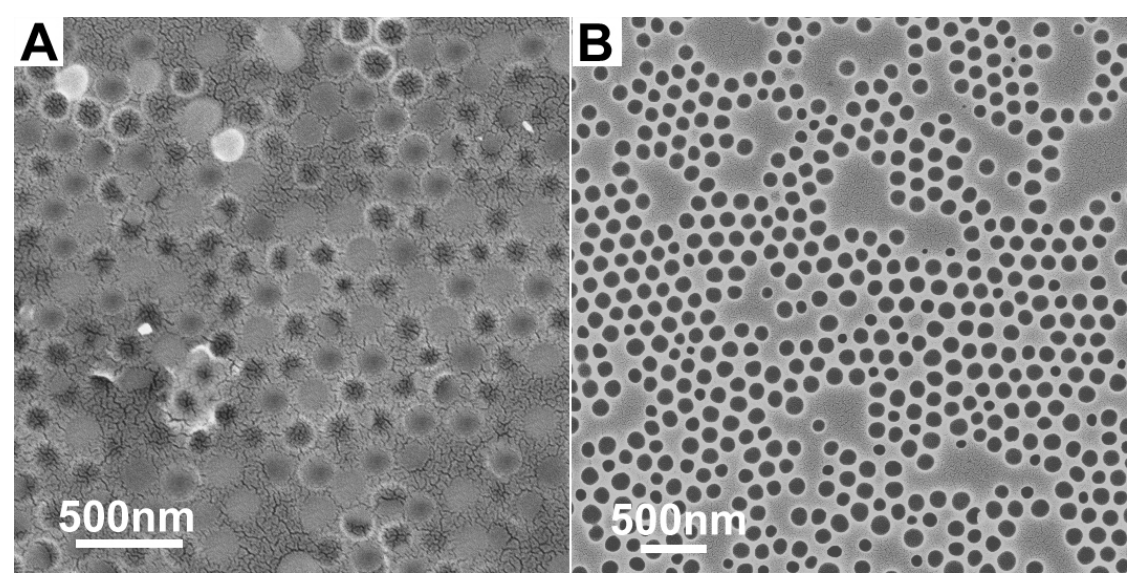

Figure S6: SEM images of the structures formed by dropping different amount of THF on the colloidal sphere array surfaces: (A) $30 \mu \mathrm{THF}$ per $0.5 \mathrm{~cm}^{2}$, (B) $100 \mu \mathrm{THF}$ per $0.5 \mathrm{~cm}^{2}$. 